\title{
Brief Insights into Zika-Microcephaly Mechanism
}

Santos GM*, Silva ITG and Amato AA

Department of Pharmacy, University of Brasilia, Brasilia, Brazil

*Corresponding author: Santos GM, Laboratory of Molecular Pharmacology, Department of Pharmacy, University of Brasília, Brasília, Brazil, Tel: 55 61 81128298; E-mail: gsantos@unb.br

Received date: February 23, 2017; Accepted date: March 24, 2017; Published date: March 31, 2017

Copyright: ( 2017 Santos GM, et al. This is an open-access article distributed under the terms of the Creative Commons Attribution License, which permits unrestricted use, distribution, and reproduction in any medium, provided the original author and source are credited.

\begin{abstract}
After recent reports attesting a straight correlation between Zika infection and brain disorders in new-borns, research questions now focus on establishing causality and the mechanisms underlying it. Studies involving monolayer cultures, murine and human brain tissue slices and the cerebral organoid system have provided important information regarding neuronal damage, but the precise mechanisms underlying neuronal cell tropism and cell damage have not been elucidated. Herein, we discuss the possibility that Zika virus proteins enter the nucleus by a brief search for nuclear signal localization and for potential nucleosome binding motifs using a bioinformatics approach and point to other questions that should be the focus of research aiming to understand Zika-virus associated cell-damage.
\end{abstract}

Keywords: Zika; Mechanism; Neuronal disorders; Microcephaly

\section{Introduction}

Great attention has recently been given to an old and forgotten virus, Zika, which was first isolated in 1947 from non-human primates in Uganda [1]. Zika virus disease was uncommon for decades; however, the uncontrolled population of Aedes aegypti mosquito has been the main culprit for spreading Zika, Dengue and Chikungunya through Brazil [2,3], other Latin American countries and Caribbean more recently [4]. Although Zika symptoms are mild or even absent in most cases, the populations from these countries have been in turmoil due to the potential link between Zika, microcephaly and other fetal malformations.

In August last year, the relationship between Zika virus and microcephaly was found improbable. Soon after, several reports associating Zika virus infection to neurological disorders started to scare the medical community. Dr. Van der Linden Mota, a Brazilian pediatrician, was the first to propose this association when dealing with a case in which a woman describing mild symptoms of Zika infection during pregnancy gave birth to twins, one with microcephaly and the other apparently healthy. This divergence between the twins in addition to the normal results from routine tests to investigate possible causes of microcephaly led Dr. Van Linden Mota to suspect she was facing a new disease.

\section{Zika Virus Infection and Neuronal Disorders}

More recently, data from several epidemiological [5,6] and preclinical [7-9] research studies supported this idea, presenting direct evidences for the association between Zika virus infection and fetal disorders, including microcephaly. In a study led in Rio de Janeiro, Brazil, fetal analysis by ultrasonography of 42 infected pregnant women showed that 12 presented fetal abnormalities (29\%). These included not only microcephaly (4 cases) but also other central nervous system (CNS) abnormalities and even fetal death. Interestingly, three of the 4 fetus in which microcephaly was detected by ultrasonography were delivered by the end of the study and microcephaly as an isolated finding was confirmed in only one of them. The other two infants were small for gestational age and head circumference was proportional to body size.

A study addressing the physiopathology of Zika virus-associated microcephaly using cell culture suggested the virus efficiently infects neural progenitor cells [10]. This was confirmed by data from research involving brain organoids [7-11] and also fetal human tissue [12,13]. In addition, it was shown that infection reduced the size of forebrain organoids by suppressing proliferation of neuronal progenitor cells and inducing apoptosis of this cell type and also of uninfected neurons $[9,10]$.

Much is understood about how flaviviruses, such as Zika, Dengue, and Yellow Fever virus, infect cells, but the exact physiopathological mechanisms underlying cell damage are not completely elucidated. Recently, it was observed that a protein highly expressed in neural stem cells, AXL, could be the main culprit for providing this type of cells with selective susceptibility to viral infection [14]. Nevertheless, it is not clear how Zika virus reaches the fetal brain, although it is reasonable to speculate this occurs by haematogenous spread after transplacental transfer.

\section{Mechanism of action of Zika virus}

Seminal work done in 1971 showed that mice infected via intracranial injection with Zika virus presented neuronal necrosis and inflammation [15]. Nevertheless, it seems that mice are not an ideal model system for neuronal studies since they differ from humans during brain development.

Zika virus, as a typical flavivirus, contains a single strand RNA genome of about 11,000 base pairs complexed with multiple copies of the capsid protein, surrounded by an icosahedral shell of both the envelope glycoprotein, with around 500 amino acids, and 75 amino acids of the membrane protein or 165 amino acids of the precursor membrane. Moreover, it has seven non-structural proteins that are important for replication, assembly, and control the host response to 
infection. Interestingly, a secondary structure constituted by a loop is degraded to form a sub genomic RNA structure, which would be crucial for pathogenicity [16]. This sub genomic RNA seems to be responsible for regulating the cellular cytosolic receptor RIG-I that recognizes viral RNA. This signaling pathway would be similar to the one adopted by Dengue virus [17].

Very recently, the structure at high resolution of Zika virus was elucidated by cryo-EM [18]. It revealed that the mature Zika virus structure was similar to mature Dengue virus and West Nile Virus structures. However, the differences in envelope glycoprotein structure between Zika virus and other flaviviruses may be crucial for dictating cellular tropism and disease outcome [19].

Taken together, the existing evidence points out that Zika virus might directly infect neuronal cells or use an indirect mechanism by which viral molecules could interfere with brain development. Indeed, a very recent report elegantly showed that Zika virus can infect human neural progenitor cells [10]. Importantly, Zika infection seems to perturb cell-cycle and activate caspase-3 to lead to cell death [10]. Moreover, Zika virus may alter global gene expression, notably down regulating genes involved in cell-cycle pathways and up regulating genes involved in transcription, protein transport and catabolic process [10]. It would be very interesting to observe which viral factors are directly involved in modulating host transcriptional events.

Some DNA viruses, such as Kaposi's sarcoma-associated Herpes virus (KSHV), may tether their genetic material to host chromatin, keeping their genome in the nuclei during cell cycle. Not only DNA viruses, but also another group of retroviruses are able to use host chromatin to surrender their genome into nuclei during cell division. In this way, viruses take the opportunity of the moment when the nuclear membrane is disrupted, avoiding the necessity of interaction with nuclear import proteins. This strategy may lead to drastic cellular consequences due viral interference with host chromosome segregation and genome maintenance [20].

From these observations, fundamental questions arise. Can Zika virus, a flavivirus, use unstructured proteins to tether host chromatin via the nuclesome surface, like other viral peptides such as the LANA peptide from Kaposi's Sarcoma-associated Herpes virus [21] and Cytomegalovirus (CMV) [22]?

It was reported that a Dengue virus capsid protein may bind histones and inhibit the genesis of nucleosomes [23]. In addition, capsid C, a structural protein from Dengue virus, colocalizes with histones in the nucleus and cytoplasm of liver cells. In vitro studies demonstrated that capsid $\mathrm{C}$ may bind to $\mathrm{H} 2 \mathrm{~A}, \mathrm{H} 2 \mathrm{~B}, \mathrm{H} 3$ and $\mathrm{H} 4$ in solution, forming heterodimers, and that this process would inhibit nucleosome formation [23]. It is notable that Dengue virus $\mathrm{C}$ protein has three nuclear localization signals (NLS), which would be the responsible to take the protein to the nucleus.

In the case for Zika virus, it would be very important to understand how Zika virus proteins are imported to the nucleus and to identify its interaction with host nuclear proteins. In order to verify whether Zika virus proteins could spend some time in the nucleus, we did a brief bioinformatics exercise searching for nuclear signal localization (NLS) in these proteins. Using NucPred program [24], we found a score of 0.4 for Zika virus polyprotein. Despite the low score (range 0-1), this does not exclude the possibility of Zika virus proteins being transported to the nucleus via interaction with endogenous proteins. Interestingly, the analyses done with protein $\mathrm{C}$ from Dengue virus did also not show a high score (0.48), however it emphasized three positively influencing subsequence indicating the potential to go to the nucleus.

In another analysis, based on the sequence of Nucleosome Binding Proteins (NBPs) with available atomic structures, we searched for potential nucleosome binding motifs in Zika virus proteins. It showed low similarity with different analysed viral NBPs, such as LANA and CMV. However, we cannot exclude the possibility that these proteins use unidentified regions on the nucleosome surface as docking sites.

\section{Conclusion}

Even with several evidences pointing to the association of Zika virus infection and neuronal disorders, it is still difficult to understand why some infected patients manifest such disorders and others do not. How could we explain the twin's case, in which one new-born had microcephaly and the other was apparently normal? Also, what could explain the regional differences in the clinical manifestations associated with fetal Zika virus infections, such as the higher rates of microcephaly in Brazil when compared with other affected countries? Is Zika virus the only culprit for brain defects or would other Aedes aegyptiborne virus, such as Dengue virus, also play a role? In addition to the still obscure mechanism of Zika virus-related tissue damage, many questions concerning the infection are still to be answered. Eliminating the vector and vaccine development are the main strategies to deal with this disease. However, understanding how the virus acts may help to develop new treatment and follow-up protocols for the vulnerable babies.

\section{References}

1. Dick GW, Kitchen SF, Haddow AJ (1952) Zika virus (I) Isolations and serological specificity. Trans R Soc Trop Med Hyg 46: 509-520.

2. Castanha PM, Cordeiro MT, Martelli CM, Souza WV, Marques ET, et al. (2013) Force of infection of dengue serotypes in a population-based study in the northeast of Brazil. Epidemiol Infect 141: 1080-1088.

3. Brasil P, Pereira JP, Moreira ME, Ribeiro Nogueira RM, Damasceno L, et al. (2016) Zika Virus Infection in Pregnant Women in Rio de Janeiro. N Engl J Med 375: 2321-2334.

4. Gyawali N, Bradbury RS, Taylor-Robinson AW (2016) The global spread of Zika virus: is public and media concern justified in regions currently unaffected? Infect Dis Poverty 5: 37.

5. Magalhães-Barbosa MC, Prata-Barbosa A, Robaina JR, Raymundo CE, Lima-Setta F (2016) Trends of the microcephaly and Zika virus outbreak in Brazil, January-July 2016. Travel Med Infect Dis 14: 458-463.

6. Bhatnagar J, Rabeneck DB, Martines RB, Reagan-Steiner S, Ermias Y, et al. (2017) Zika Virus RNA Replication and Persistence in Brain and Placental Tissue. Emerg Infect Dis 23: 405-414.

7. Cugola FR, Fernandes IR, Russo FB, Freitas BC, Dias JL, et al. (2016) The Brazilian Zika virus strain causes birth defects in experimental models. Nature 534: 267-271.

8. Garcez PP, Loiola EC, Madeiro da Costa R, Higa LM, Trindade P, et al. (2016) Zika virus impairs growth in human neurospheres and brain organoids. Science 352: 816-818.

9. Qian X, Nguyen HN, Song MM, Hadiono C, Ogden SC, et al. (2016) Brain-Region-Specific Organoids Using Mini-bioreactors for Modeling ZIKV Exposure. Cell 165: 1238-1254.

10. Tang H, Hammack C, Ogden SC, Wen Z, Qian X, et al. (2016) Zika Virus Infects Human Cortical Neural Progenitors and Attenuates Their Growth Brief Report Zika Virus Infects Human Cortical Neural Progenitors and Attenuates Their Growth. Cell Stem Cell 18: 587-590.

11. Dang J, Tiwari SK, Lichinchi G, Qin Y, Patil VS, et al. (2016) Zika Virus Depletes Neural Progenitors in Human Cerebral Organoids through 
Citation: Santos GM, Silva ITG, Amato AA (2017) Brief Insights into Zika-Microcephaly Mechanism. Clin Exp Pharmacol 7: 235. doi: 10.4172/2161-1459.1000235

Page 3 of 3

Activation of the Innate Immune Receptor TLR3. Cell Stem Cell 19: 258-265.

12. Onorati M, Li Z, Liu F, Sousa AM, Nakagawa N, et al. (2016) Zika Virus Disrupts Phospho-TBK1 Localization and Mitosis in Human Neuroepithelial Stem Cells and Radial Glia. Cell Rep 16: 2576-2592.

13. Retallack H, Di Lullo E, Arias C, Knopp K, Laurie MT, et al. (2016) Zika virus cell tropism in the developing human brain and inhibition by azithromycin. Proc Natl Acad Sci USA, 113: 14408-14413.

14. Nowakowski TJ, Pollen AA, Di Lullo E, Sandoval-Espinosa C, Bershteyn M, et al. (2016) Expression Analysis Highlights AXL as a Candidate Zika Virus Entry Receptor in Neural Stem Cells. Cell Stem Cell 18: 591-596.

15. Bell TM, Field EJ, Narang HK (1971) Zika virus infection of the central nervous system of mice. Arch Gesamte Virusforsch 35: 183-193.

16. Clarke BD, Roby JA, Slonchak A, Khromykh AA (2015) Functional noncoding RNAs derived from the flavivirus 3' untranslated region. Virus Res 206: 53-61.

17. Manokaran G, Finol E, Wang C, Gunaratne J, Bahl J, et al. (2015) Dengue subgenomic RNA binds TRIM25 to inhibit interferon expression for epidemiological fitness. Science 350: 217-221.

18. Sirohi D, Chen Z, Sun L, Klose T, Pierson TC, et al. (2016) The $3.8 \AA$ resolution cryo-EM structure of Zika virus. Science 5316: 1-7.
19. Brault JB, Khou C, Basset J, Coquand L, Fraisier V, et al. (2016) Comparative Analysis Between Flaviviruses Reveals Specific Neural Stem Cell Tropism for Zika Virus in the Mouse Developing Neocortex. EBioMedicine, The Ohio State University Wexner Medical Center 10: 71-76.

20. Aydin I, Schelhaas M (2016) Viral genome tethering to host cell chromatin: cause and consequences. Traffic 17: 327-340.

21. Barbera AJ, Chodaparambil JV, Kelley-Clarke B, Joukov V, Walter JC, et al. (2006) The nucleosomal surface as a docking station for Kaposis sarcoma herpesvirus LANA. Science 311: 856-861.

22. Fang Q, Chen P, Wang M, Fang J, Yang N, et al. (2016) Human cytomegalovirus IE1 protein alters the higher-order chromatin structure by targeting the acidic patch of the nucleosome. Elife 5 .

23. Colpitts TM, Barthel S, Wang P, Fikrig E (2011) Dengue virus capsid protein binds core histones and inhibits nucleosome formation in human liver cells. PLoS ONE 6: e24365.

24. Brameier M, Krings A, MacCallum RM (2007) NucPred-Predicting nuclear localization of proteins. Bioinformatics 23: 1159-1160. 